PESQUIMAT, Revista de la F.C.M. de la

Universidad Nacional Mayor de San Marcos

Vol. XIV N¹, pp. 22-33, Lirna - Perú, Julio 2011

\title{
LA TRANSFORMADA DE ZAK
}

\begin{abstract}
Luis Miguel Nuñez Ramirez ${ }^{*}$, Humberto Emiliano Galvez Perez ${ }^{* *}$ \& Rodolfo José Galvez Perez ${ }^{* * *}$
Resumen: La Transformada de Zak, es una técnica que juega un rol importante en el estudio del problema de la representación de Gabor en el análisis de señales. La Transformada de Zak de una señal $f$ puede ser considerada como una generalización de su Transformada de Fourier discreta; se estudia algunas de sus propiedades, así como su aplicación en la demostración del Teorema de Balian-Low el cual es un resultado fundamental en el análisis tiempo-frecuencia. Palabras clave: Transformada de Zak, Base Ortonormal, Frame, Teorema de Balian-Low.
\end{abstract}

\section{ZAK TRANSFORM}

Abstract: The Zak transform is a technique that plays an important role in studying the problem of Gabor representation in signal analysis. The Zak transform of a signal $f$ can be considered as a generalization of the discrete Fourier transform. we study some properties and its application in demonstrating the Balian-Low theorem which is a fundamental result in the analysis time-frequency.

Key words: Zak transform. Ortonormal Basis, Frame, Balian-Low Theorem.

\section{Introducción}

Históricamente, la Transformada de Zak, conocido como la transformada de Weil-Brezin en el análisis armónico. fue presentado por Gelfand (1950) en su famoso artículo sobre la expansión de funciones propias asociadas a los operadores de Schrödinger con potencial periódico. Esta transformación tambión era conocida como la aplicación de Gelfand en la literatura matemática rusa. Sin embargo, Zak $(1967,1968)$ independientemente la redescubrió como la Tranformada k-q. en física del estado sólido para el estudio de la mecánica cuántica del movimiento de los electrones en presencia de un campo eléctrico o magnético. En los últimos años, la Transformada de Zak ha sido ampliamente utilizado en el análisis tiempofrecuencia de una señal, así como en el análisis matemático de los sistemas de Gabor. En particular, la Transformada de Zak también ha sido útil para el estudio del problema de la representación Gabor. donde esta transformación ha sido utilizado con éxito para investigar la ortogonalidad y la integridad de los frames de Gabor en el caso crítico.

\section{La Transformada de Zak}

Definición.Sea $a \in \mathbb{R}, a>0$, la Transfomada de Zak de una función $f$ se denota como $Z f$ y se define por

$$
Z f(t, w)=\sqrt{a} \sum_{k} f(t a+k a) e^{2 \pi i k w}
$$

donde $t . w$ son reales.

\footnotetext{
*UNMSMS, Facultad de Ciencias Matemáticas, e-mail: lnuñezr(unmsm.edu.pe

**NASMS, Facultad de Ciencias Matemáticas, e-mail: hgalvezp@ummsm.edu.pe

*** UNMSMS, Facultad de Ciencias Matemáticas, e-mail: hgalvezp@ìmmsm.edu.pe
} 
Si $\sum_{k}|f(t a+k a)|<\infty$ entonces $Z f(t, w)$ es convergente, otro criterio para la convegencia es asumir que $|f(t a+k a)| \leq \frac{c}{(1+|t|)^{1+\varepsilon}}, \varepsilon>0$

\section{Corolário 1.}

La Transfomada de Zak cumple las propiedades de cuasiperiodicidad, es decir:

i) $Z f(t+1, w)=Z f(t, w) e^{-2 \pi i w}$

ii) $Z f(t, w+1)=Z f(t, w)$

\section{Demostración.}

En efecto,

$$
\begin{aligned}
Z f(t+1, w) & =\sum_{k} f((t+1) a+k a) e^{2 \pi i k w} \\
& =\sum_{k} f(t a+(k+1) a) e^{2 \pi i k w} \\
& =\sum_{k} f(t a+(k+1) a) e^{2 \pi i(k+1) w} e^{-2 \pi i w} \\
& =Z f(t, w) e^{-2 \pi i w}
\end{aligned}
$$

Asimismo

$$
\begin{aligned}
Z f(t, w+1) & =\sum_{k} f(t a+k a) e^{2 \pi i k(w+1)}=\sum_{k} f(t a+k a) e^{2 \pi i k w} e^{2 \pi i k} \\
& =\sum_{k} f(t a+k a) e^{2 \pi i k w}=(Z f)(t, w)
\end{aligned}
$$

El siguiente corolário establece que es suficiente trabajar con la Transformada de Zak en el cuadrado unitario.

\section{Corolário 2 .}

Sea $\mathbb{L}^{2}(\mathbb{Q})=\left\{F: \mathbb{Q} \longrightarrow \mathbb{C}(\dot{o} \mathbb{R}) / \int_{0}^{1} \int_{0}^{1}|F(t, w)|^{2} d t d w^{\prime}<\infty\right\}$.

$\mathbb{L}^{2}(\mathbb{Q})$ es un espacio de Hilbert con producto interno

$$
\langle F, G\rangle_{\mathbb{L}^{2}(\mathbb{Q})}=\int_{0}^{1} \int_{0}^{1} F(t, w) \overline{G(t, w)} d t d w
$$

y norma

$$
\|F\|_{\mathbb{L}^{2}(Q)}^{2}=\int_{0}^{1} \int_{0}^{1}|F(t, w)|^{2} d t d w
$$

\section{Demostración.}

Esto se prueba verificando que si $\left\|F_{n}-F_{m}\right\|_{\mathbb{L}^{2}(\mathbb{Q})} \longrightarrow 0$ cuando $n, m \rightarrow \infty$, entonces existe $F \in \mathbb{L}^{2}(\mathbb{Q})$ tal que $F_{n} \rightarrow F$ cuando $n \rightarrow \infty$.

\section{Observación:}

1.- Sea $\mathbb{H}_{1}=\mathbb{L}^{2}([0,1))$. $\mathbb{H}_{2}=\mathbb{L}^{2}([0,1))$, si $\left\{e^{2 \pi i m t}\right\}$ es una base ortonormal para $\mathbb{H}_{1}=\mathbb{L}^{2}([0,1))$ y $\left\{e^{2 \pi i n w}\right\}$ es una base ortonormal para $\mathbb{H}_{2}=\mathbb{L}^{2}([0.1))$. entonces $\left(e^{2 \pi i m t} \cdot e^{2 \pi i n u}\right)$ es una base ortonormal para $\mathbb{H}_{1} \times \mathbb{H}_{2}=\mathbb{L}^{2}([0,1)) \times \mathbb{L}^{2}([0,1))=\mathbb{L}^{2}(\mathbb{Q})$. Por lo tanto $E_{m, n}=E_{m, n}(t, w)=e^{2 \pi i m t} e^{2 \pi i n w}$ es una base ortonormal para $\mathbb{L}^{2}(\mathbb{Q})$.

2.- La aplicación

$$
\mathbb{L}^{2}(\mathbb{R}) \underset{f}{\longrightarrow} \mathbb{L}^{2}(\mathbb{R})
$$

es un isomorfismo isométrico y además $\|f\|_{\mathbb{L}^{2}(\mathbb{K})}=\frac{1}{2 \pi}\|\hat{f}\|_{\mathbb{L}^{2}(\mathbb{R})}$.

Para el signiente resultado recordemos que si $\mathbb{H}_{1}$ y $\mathbb{H}_{2}$ son espacios de Hillert, la aplicación $U: \mathbb{H}_{1} \longrightarrow \mathbb{H}_{2}$ es un operador unitario, si satisface: 
i) $\langle U v, U w\rangle=\langle v, w\rangle \forall v, w \in \mathbb{H}_{1}$

ii) $U \mathbb{H}_{1}=\mathbb{H}_{2}$, esto es, para cada $v_{2} \in \mathbb{H}_{2}, v_{2}=U v_{1}$, para algún $v_{1} \in \mathbb{H}_{1}$.

Además si $\left\{e_{n}\right\}$ es una base en $\mathbb{H}_{1},\left\{U e_{n}\right\}$ es una base en $\mathbb{H}_{2}$ y reciprocamente.

\section{Teorema 3.}

La Transformada de Zak es un operador unitario de $\mathbb{L}^{2}(\mathbb{R})$ sobre $\mathbb{L}^{2}(\mathbb{Q})$, y además $\|Z f\|_{\mathbb{L}^{2}(\mathbb{Q})}=\|f\|_{\mathbb{L}^{2}(\mathbb{R})}$. Demostración.

Sea $f \in \mathbb{L}^{2}(\mathbb{R}) ; Z f(t, w)=\sqrt{a} \sum_{k} f(t a+k a) e^{2 \pi i k w}$, haciendo

$$
F_{k}(t, w)=\sqrt{a} f(t a+k a) e^{2 \pi i k w}
$$

entonces

$$
Z f(t, w)=\sum_{k} F_{k}(t, w)
$$

Afirmación 1: $F_{k} \in \mathbb{L}^{2}(\mathbb{Q})$

En efecto,

$$
\begin{aligned}
\left\|F_{k}\right\|_{\mathbb{L}^{2}(\mathbb{Q})}^{2}= & a \int_{0}^{1} \int_{0}^{1}|f(t a+k a)|^{2}\left|e^{2 \pi i k w}\right|^{2} d t d w \\
= & a \int_{0}^{1} \int_{0}^{1}|f(t a+k a)|^{2} d t d w=a \int_{0}^{1}|f(t a+k a)|^{2} d t\left(\int_{0}^{1} d w\right) \\
& (\text { debido al teorema de Fubini) } \\
= & a \int_{0}^{1}|f(t a+k a)|^{2} d t
\end{aligned}
$$

hacemos

$$
\begin{aligned}
\tilde{t} & =t a \Rightarrow d \widetilde{t}=a d t \\
t & =0 \Rightarrow \widetilde{t}=0 \\
t & =1 \Rightarrow \widetilde{t}=a,
\end{aligned}
$$

se tiene

$$
\begin{aligned}
\left\|F_{k}\right\|_{\mathbb{L}^{2}(\mathbb{Q})}^{2} & =a \int_{0}^{a}|f(\widetilde{t}+k a)|^{2} \frac{d \widetilde{t}}{a}=\int_{0}^{a}|f(\widetilde{t}+k a)|^{2} d \widetilde{t} \\
& =\int_{0}^{a}|f(t+k a)|^{2} d t \leq \int_{-\infty}^{+\infty}|f(t+k a)|^{2} d t \\
& \leq \int_{-\infty}^{+\infty}|f(t)|^{2} d t=\|f\|_{\mathbb{L}^{2}(\mathbb{R})}^{2}<\infty .
\end{aligned}
$$

Por lo tanto

$$
\left\|F_{k}\right\|_{\mathbb{L}^{2}(\mathbb{Q})}^{2}<\infty, \text { esto es, } F_{k} \in \mathbb{L}^{2}(\mathbb{Q}) .
$$

Afirmación 2: $\left\{F_{k}\right\}_{k \in Z}$ es ortogonal .

En efecto,

$$
\begin{aligned}
\left\langle F_{k}, F_{m}\right\rangle_{\mathbb{L}^{2}(\mathbb{Q})}= & \left\langle\sqrt{a} f(t a+k a) e^{2 \pi i k w}, \sqrt{a} f(t a+m a) e^{2 \pi i m w}\right\rangle_{\mathbb{L}^{2}(\mathbb{Q})} \\
= & a \int_{0}^{1} \int_{0}^{1} f(t a+k a) e^{2 \pi i k w} \overline{f(t a+m a)} e^{-2 \pi i m w} d t d w \\
& (\text { debido al teorema de Fubini) } \\
= & a \int_{0}^{1}\left[\int_{0}^{1} e^{2 \pi i k w} e^{-2 \pi i m w} d w\right] f(t a+k a) \overline{f(t a+m a)} d t
\end{aligned}
$$

como $\left\{e^{2 \pi i k w}\right\}_{k \in \mathbb{Z}}$ es ortogonal, entonces $\int_{0}^{1} e^{2 \pi i k w} e^{-2 \pi i m w} d w=0$ 
Por lo tanto

$$
\left\langle F_{k}, F_{m}\right\rangle_{\mathbb{L}^{2}(\mathbb{Q})}=0
$$

esto es, $\left\{F_{k}\right\}$ es ortogonal.

Afirmacion 3: $\|Z f\|_{\mathbb{L}^{2}(\mathbb{Q})}=\|f\|_{\mathbb{L}^{2}(\mathbb{R})}$

En efecto,

$$
\begin{aligned}
\|Z f\|_{\mathbb{L}^{2}(\mathbb{Q})} & =\left\|\sum_{k} F_{k}\right\|_{\mathbb{L}^{2}(\mathbb{Q})}^{2}=\left\langle\sum_{k} F_{k}, \sum_{m} F_{m}\right\rangle_{\mathbb{L}^{2}(\mathbb{Q})} \\
& =\sum_{k} \sum_{m}\left\langle F_{k}, F_{m}\right\rangle_{\mathbb{L}^{2}(\mathbb{Q})}=\sum_{k}\left\langle F_{k}, F_{k}\right\rangle_{\mathbb{L}^{2}(\mathbb{Q})} \\
& =\sum_{k}\left\|F_{k}\right\|^{2}=\sum_{k} \int_{0}^{a}|f(t+k a)|^{2} d t
\end{aligned}
$$

hacemos

$$
\begin{aligned}
\widetilde{t} & =t+k a \Rightarrow d \widetilde{t}=d \tilde{t} \\
t & =0 \Rightarrow \widetilde{t}=k a \\
t & =a \Rightarrow \widetilde{t}=(k+1) a,
\end{aligned}
$$

luego

$$
\begin{aligned}
\|Z f\|_{\mathbb{L}^{2}(\mathbb{Q})} & =\sum_{k} \int_{k: a}^{(k+1) a}|f(\widetilde{t})|^{2} d \widetilde{t}=\sum_{k} \int_{k a \cdot}^{(k+1) a}|f(t)|^{2} d t \\
& =\int|f(t)|^{2} d t=\|f\|_{\mathbb{L}^{2}(\mathbb{R})}
\end{aligned}
$$

Por lo tanto

$$
\|Z f\|_{\mathbb{L}^{2}(\mathbb{Q})}=\|f\|_{\mathbb{L}^{2}(\mathbb{R})}
$$

\section{Corolário 4.}

Se tiene que $E_{m, n}=E_{m, n}(t, w)=e^{2 \pi i m t} e^{2 \pi i n w}$ es una base ortonormal para $\mathbb{L}^{2}(\mathbb{Q})$.

Demostración.

Sea

$$
\begin{aligned}
e_{m, n}(x) & =a^{-1 / 2} T_{n a} E_{\frac{m}{a}} \chi_{[0, a)}(x), a>0 \\
& =a^{-1 / 2} e^{2 \pi i \frac{m}{a}(x-n a)} \chi_{[0, a)}(x-n a)
\end{aligned}
$$

hacemos

$$
x-n a=\bar{x} ; 0 \leq x-n a<a \Leftrightarrow n a \leq x<(n+1) a \Leftrightarrow x \in[n a,(n+1) a)
$$

luego

$$
e_{m, n}(x)=a^{-1 / 2} e^{2 \pi i \frac{m}{a} \bar{x}} \lambda_{[n a \cdot(n+1) a)}(\bar{x})
$$

esto es

$$
\left\{c_{m, n}(x)\right\}_{m, n \in \mathbb{Z}}=\left\{a^{-1 / 2} e^{2 \pi i \frac{m}{n} x} \gamma_{[m a,(n+1) a)}(x)\right\}_{m, n \in \mathbb{Z}}
$$

por tanito $\left\{e_{m, n}(x)\right\}_{m, n \in \mathbb{Z}}$ (s una base ortonormal para $\mathbb{L}^{2}(\mathbb{R})$.

Afirmación: $Z e_{m, n}(t, w)=E_{m, n}(t, w)$

En efecto,

$$
\begin{aligned}
Z e_{m, n}(t, w) & =\sum_{k} e_{m, n}(t a+k a) e^{2 \pi i k w} \\
& =\sum_{k} a^{1 / 2} a^{-1 / 2} e^{2 \pi i \frac{m}{a}(t a+k a)} \chi_{[n a,(n+1) a)}(t a+k a) e^{2 \pi i k w}
\end{aligned}
$$

hacemos

$$
\begin{aligned}
t a+x a & =\tilde{t} ; n a \leq t a+k a<(n+1) a \Leftrightarrow n a-k a \leq t a<(n+1) a-k a \\
& \Leftrightarrow n-k \leq t<(n+1)-k \Leftrightarrow n-k \leq t<(n-k)+1
\end{aligned}
$$


luego

$$
\begin{aligned}
Z e_{m, n}(t, w) & =\sum_{k} e^{2 \pi i m t} e^{2 \pi i m k} e^{2 \pi i k w} \chi_{[n-k, n-k+1)}(\widetilde{t}) \\
& =e^{2 \pi i m t} \sum_{k} e^{2 \pi i k w} \chi_{[n-k, n-k+1)}(t), \text { donde } t \in[0,1)
\end{aligned}
$$

Entonces

$$
\begin{aligned}
\left(Z e_{m, n}\right)(t, w)= & e^{2 \pi i m t}\left[\ldots \ldots \ldots+e^{2 \pi i(n-1) w} \chi_{[1,2)}(t)+e^{2 \pi i n w} \chi_{[0,1)}(t)+\right. \\
& \left.+e^{2 \pi i(n+1) w} \chi_{[-1,0)}(t)+\ldots \ldots . .\right] \\
= & e^{2 \pi i m t} e^{2 \pi i n t}=E_{m, n}(t, w)
\end{aligned}
$$

\section{Corolário 5 .}

Sean $a, b>0$. Si $a \cdot b=1$,entonces

$$
Z\left(E_{m b} T_{n a} g\right)(t, w)=Z\left(g_{m b, n a}\right)(t, w)=E_{m, n}(t, w)(Z g)(t, w) \text { ó } Z\left(g_{m b, n a}\right)=E_{m, n}(Z g)
$$

\section{Demostración.}

En efecto,

$$
\begin{aligned}
Z\left(E_{m b} T_{n a} g\right)(t, w)= & \sum_{k} a^{1 / 2} E_{m b} T_{n a} g(t a+k a) e^{2 \pi i k w} \\
= & \sum_{k} a^{1 / 2} e^{2 \pi i m b(t a+k a)} g(t a+k a-n a) e^{2 \pi i k w} \\
= & \sum_{k} a^{1 / 2} e^{2 \pi i m b t a} e^{2 \pi i m b k a} g(t a+(k-n) a) e^{2 \pi k w} \\
= & \sum_{k} a^{1 / 2} e^{2 \pi i(k-n) w} g(t a+(k-n) a) e^{2 \pi i n w} e^{2 \pi i m t} e^{2 \pi i m k}, \\
& (\text { debido a la hipótesis) } \\
= & \left(e^{2 \pi i m t} e^{2 \pi i n w}\right)\left(\sum_{k} a^{1 / 2} e^{2 \pi i(k-n) w} g(t a+(k-n) a) e^{2 \pi i m k}\right) \\
= & \left(e^{2 \pi i m t} e^{2 \pi i n w}\right)\left(\sum_{k} a^{1 / 2} e^{2 \pi i(k-n) w} g(t a+(k-n) a)\right), \text { pues } e^{2 \pi i m k}=1
\end{aligned}
$$

Luego

$$
\begin{aligned}
& Z\left(E_{m b} T_{n a} g\right)(t, w)=\left(e^{2 \pi i m t} e^{2 \pi i n w}\right)(Z g)(t, w) \\
& Z\left(E_{m b} T_{n a} g\right)(t, w)=E_{m, n}(t, w)(Z g)(t, w)
\end{aligned}
$$

Por lo tanto

$$
Z\left(g_{m b, n a}\right)=E_{m, n}(Z g)
$$

\section{Aplicaciones de la Transformada de Zak}

\section{Teorema 6.}

Sean $a, b>0$ tal que $a b=1$ y $g \in \mathbb{L}^{2}(\mathbb{R})$. Entonces son equivalentes las siguientes afirmaciones:

i) Existen $A, B$ constantes tales que $0<A \leq|Z g|^{2} \leq B<\infty$.

ii) $\left\{g_{m b, n a}(x)\right\}=\left\{e^{2 \pi i m b x} g(x-n a)\right\}$ es un frame para $\mathbb{L}^{2}(\mathbb{R})$ con cotas frames $A$ y $B$.

iii) $\left\{g_{m b, n a}\right\}$ es un frame exacto para $\mathbb{L}^{2}(\mathbb{R})$ con cotas frames $A, B$ y además para cualquier $f$ $\in \mathbb{L}^{2}(\mathbb{R}), f=\sum_{m, n} C_{m, n} g_{m b ; n a}$, donde

$$
C_{m, n}=\int_{0}^{1} \int_{0}^{1} \frac{(Z f)(t, w)}{(Z g)(t, w)} e^{-2 \pi i m t} e^{-2 \pi i n w} d t d w
$$




\section{Demostración.}

i) $\Rightarrow$ ii) Sea $a, b>0, a b=1$

$$
\begin{aligned}
& \mathbb{L}^{2}(\mathbb{R}) \stackrel{\text { Unitario }}{\longmapsto} \mathbb{L}^{2}(\mathbb{Q}) \\
& g_{m b, n a} \longmapsto Z g_{m b, n a} \\
&\left\|g_{m b, n a}\right\|_{\mathbb{L}^{2}(\mathbb{R})}=\left\|Z g_{m b, n a}\right\|_{\mathbb{L}^{2}(\mathbb{Q})}=\left\|E_{m, n} Z g\right\|_{\mathbb{L}^{2}(\mathbb{Q})}=\|Z g\|_{\mathbb{L}^{2}(\mathbb{Q})}=\|g\|_{\mathbb{L}^{2}(\mathbb{R})}
\end{aligned}
$$

Deseamos probar que, si $\left\{g_{m b, n a}\right\}$ es un frame para $\mathbb{L}^{2}(\mathbb{R})$ si y sólo si $\left\{Z g_{m b, n a}\right\}$ es un frame para $\mathbb{L}^{2}(\mathbb{Q})$. En efecto, sea $F \in \mathbb{L}^{2}(\mathbb{Q})$,tenemos que

$$
\begin{aligned}
\left\langle F, Z g_{m b, n a}\right\rangle & =\left\langle F, E_{m, n}(Z g)\right\rangle=\int_{0}^{1} \int_{0}^{1} F \overline{E_{m, n}(Z g)} d t d w=\int_{0}^{1} \int_{0}^{1} F \overline{(Z g)} \overline{E_{m, n}} d t d w \\
& =\left\langle F \overline{(Z g)}, E_{m, n}\right\rangle_{\mathbb{L}^{2}(\mathbb{Q})}
\end{aligned}
$$

por consiguiente

$$
\begin{aligned}
\sum_{m, n}\left|\left\langle F, Z g_{m b, n a}\right\rangle\right|^{2} & =\sum_{m, n}\left|\left\langle F \overline{(Z g)}, E_{m, n}\right\rangle\right|^{2}=\|F \overline{(Z g)}\|_{\mathbb{L}^{2}(\mathbb{Q})} \\
& =\int_{0}^{1} \int_{0}^{1}|F|^{2}|\overline{Z g}|^{2} d t d w=\int_{0}^{1} \int_{0}^{1}|F|^{2}|Z g|^{2} d t d w \\
& \leq B \int_{0}^{1} \int_{0}^{1}|F|^{2} d t d w=B\|F\|_{\mathbb{L}^{2}(\mathbb{Q})}^{2}<\infty
\end{aligned}
$$

debido a que $\left\{E_{m, n}\right\}$ es una base ortonormal para $\mathbb{L}^{2}(\mathbb{Q})$, y por el teorema de Parseval.

Analogamente

$$
A\|F\|_{\mathbb{L}^{2}(\mathbb{Q})}^{2}=A \int_{0}^{1} \int_{0}^{1}|F|^{2} d t d w \leq \int_{0}^{1} \int_{0}^{1}|F|^{2}|Z g|^{2} d t d w=\|F(\overline{Z g})\|_{\mathbb{L}^{2}(\mathbb{Q})}^{2}
$$

esto es

$$
A\|F\|_{\mathbb{L}^{2}(\mathbb{Q})}^{2} \leq \sum_{m, n}\left|\left\langle F, Z g_{m b, n a}\right\rangle\right|^{2} \leq B\|F\|_{\mathbb{L}^{2}(\mathbb{Q})}^{2}
$$

Por lo tanto $\left\{Z g_{m b, n a}\right\}$ es un frame, esto es, $\left\{g_{m b, n a}\right\}$ es un frame para $\mathbb{L}^{2}(\mathbb{R})$.

ii) $\Rightarrow$ i) Sabemos que $\left\{g_{m, b, n a}\right\}$ es un frame para $\mathbb{L}^{2}(\mathbb{R})$ con cotas frames $A$ y $B$ si y sólo si $\left\{Z g_{m b, n a}\right\}$ es un frame para $\mathbb{L}^{2}(\mathbb{Q})$, es decir $\left\{E_{m, n} Z g\right\}_{m, n \in \mathbb{Z}}$ es un frame para $\mathbb{L}^{2}(\mathbb{Q})$. Luego, para todo $F \in \mathbb{L}^{2}(\mathbb{Q})$ se tiene

$$
A\|F\|_{\mathbb{L}^{2}(\mathbb{Q})}^{2} \leq \sum_{m, n}\left|\left\langle F, E_{m, n}(Z g)\right\rangle\right|^{2} \leq B\|F\|_{\mathbb{L}^{2}(\mathbb{Q})}^{2}
$$

esto es,

$$
A\|F\|_{\mathbb{L}^{2}(\mathbb{Q})} \leq \sum_{m, n}\left|\left\langle F(\overline{Z g}), E_{m, n}\right\rangle\right|^{2}=\|F(\overline{Z g})\|_{\mathbb{L}^{2}(\mathbb{Q})}^{2} \leq B\|F\|_{\mathbb{L}^{2}(\mathbb{Q})}^{2}
$$

Por consiguiente

$$
\|F(\overline{Z g})\|_{\mathbb{L}^{2}(\mathbb{Q})}^{2}=\int_{0}^{1} \int_{0}^{1}|F|^{2}|Z g|^{2} d t d w \leq B \int_{0}^{1} \int_{0}^{1}|F|^{2} d t d w \leq \int_{0}^{1} \int_{0}^{1}|F|^{2} B d t d w
$$

entonces

$$
|Z g|^{2} \leq B
$$

Analogamente

$$
\int_{0}^{1} \int_{0}^{1}|F|^{2} A d t d w=A \int_{0}^{1} \int_{0}^{1}|F|^{2} d t d w \leq \int_{0}^{1} \int_{0}^{1}|F|^{2}|Z g|^{2} d t d w
$$

entonces

$$
A \leq|Z g|^{2}
$$


Por lo tanto

$$
A \leq|Z g|^{2} \leq B<\infty
$$

(ii) $\Rightarrow$ (iii) Sabemos que si $\left\{g_{m b, n a}\right\}$ es un frame exacto si y sólo si es una base de Riesz. Por hipótesis $\left\{g_{m b, n a}\right\}$ es un frame para $\mathbb{L}^{2}(\mathbb{R})$ con cotas frames $A$ y $B$, entonces $\left\{Z g_{m b, n a}\right\}$ es un frame para $\mathbb{L}^{2}(\mathbb{Q})$, esto es debido a que $Z: \mathbb{L}^{2}(\mathbb{R}) \longrightarrow \mathbb{L}^{2}(\mathbb{Q})$ es un operador unitario y $\|Z f\|_{\mathbb{L}^{2}(\mathbb{Q})}=\|f\|_{\mathbb{L}^{2}(\mathbb{R})}$.

Definimos

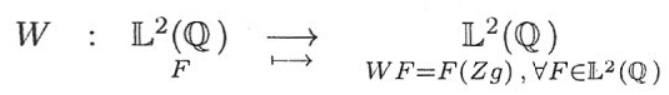

Afirmación 1: $W$ es un Isomorfismo Topológico, es decir $W$ es un Homeómorfismo lineal.

Probaremos que $W$ es continua, en efecto

$$
\begin{aligned}
\|W F\|_{\mathbb{L}^{2}(\mathbb{Q})}^{2} & =\int_{0}^{1} \int_{0}^{1}|W F|^{2}=\int_{0}^{1} \int_{0}^{1}|F(Z g)|^{2}=\int_{0}^{1} \int_{0}^{1}|F|^{2}|Z g|^{2} \\
A\|F\|_{\mathbb{L}^{2}(\mathbb{Q})}^{2} & \leq\|W F\|_{\mathbb{L}^{2}(\mathbb{Q})}^{2} \leq B\|F\|_{\mathbb{L}^{2}(\mathbb{Q})}^{2},
\end{aligned}
$$

por lo tanto, $W$ es continua.

Veamos que $W$ es inyectiva, esto es, si $W F=0$, entonces probaremos que $F=0$.

En efecto, como $A\|F\|_{\mathbb{L}^{2}(\mathbb{Q})}^{2} \leq\|W F\|_{\mathbb{L}^{2}(\mathbb{Q})}^{2}=0$ entonces $\|F\|_{\mathbb{L}^{2}(\mathbb{Q})}^{2}=0$ si y sólo si $F=0$.

Para demostrar, que $W$ es sobreyectiva, recordemos que si se tiene $(H,\langle\rangle) 1$ m espacio de Hilbert, donde $A=\left\{u_{\alpha} / \alpha \in I\right\}$ es una familia ortonormal; son equivalentes las siguientes afirmaciones:

a) $A$ es una base ortonormal de $H$.

b) Sea $x \in H$ con $x \neq 0$, entonces $x=\sum_{\alpha}\left\langle x, u_{\alpha}\right\rangle u_{\alpha}$.

c) Sea $x \in H$ con $x \neq 0$, entonces $\|x\|^{2}=\sum_{a}\left|\left\langle x, u_{\alpha}\right\rangle\right|^{2}$.(Parseval)

d) $\mathrm{Si} x \in H$ tal que $\left\langle x, u_{\alpha}\right\rangle=0$ para todo $\alpha \in I$ entonces $x=0$.

Como $\left\{E_{m, n}(t, w)\right\}$ esuna base ortonormal para $\mathbb{L}^{2}(\mathbb{Q})$. entonces

$$
\begin{aligned}
W F & =W\left(\sum\left\langle F, E_{m, n}\right\rangle E_{m, n}\right)=\sum W\left(\left\langle F, E_{m, n}\right\rangle E_{m, n}\right) \\
W F & =\sum\left\langle F \cdot E_{m, n}\right\rangle W\left(E_{m, n}\right)=\sum\left\langle F, E_{m, n}\right\rangle E_{m, n}(Z g) \\
& =\left(\sum\left\langle F, E_{m, n}\right\rangle E_{m, n}\right) Z g=F(Z g) .
\end{aligned}
$$

Por lo tanto, $W$ es sobreyectiva, luego existe $W^{-1}$. Veamos que $W^{-1}$ es continua, esto es

$$
C\|G\|_{\mathbb{L}^{2}(\mathfrak{g})}^{2} \leq\left\|W^{-1} G\right\|_{\mathbb{L}^{2}(\mathbb{Q})} \leq D\|G\|_{\mathbb{L}^{2}(\mathbb{Q})}^{2}, \forall G \in \mathbb{L}^{2}(\mathbb{Q}) .
$$

Sea $G \in \mathbb{L}^{2}(\mathbb{Q}), W^{-1}(G) \in \mathbb{L}^{2}(\mathbb{Q})$ entonces en

$$
A\|G\|_{\mathbb{L}^{2}(\mathbb{Q})}^{2} \leq\|W G\|_{\mathbb{L}^{2}(\mathbb{Q})}^{2} \leq B\|G\|_{\mathbb{L}^{2}(\mathbb{Q})}^{2}
$$

tomando $W^{-1} G$ por $G$, se tiene

$$
\begin{aligned}
A\left\|W^{-1} G\right\|_{\mathbb{L}^{2}(\mathbb{Q})}^{2} & \leq\left\|W\left(W^{-1} G\right)\right\|_{\mathbb{L}^{2}(\mathbb{Q})}^{2} \leq B\left\|W^{-1} G\right\|_{\mathbb{L}^{2}(\mathbb{Q})}^{2} \\
A\left\|W^{-1} G\right\|_{\mathbb{L}^{2}(\mathbb{Q})}^{2} & \leq\|G\|_{\mathbb{L}^{2}(\mathbb{Q})}^{2} \leq B\left\|W^{-1} G\right\|_{\mathbb{L}^{2}(\mathbb{Q})}^{2}, \text { esto es, } \\
\left\|W^{-1} G\right\|_{\mathbb{L}^{2}(\mathbb{Q})}^{2} & \leq A^{-1}\|G\|_{\mathbb{L}^{2}(\mathbb{Q})}^{2} \cdot B^{-1}\|G\|_{\mathbb{L}^{2}(\mathbb{Q})}^{2} \leq\left\|W^{-1} G\right\|_{\mathbb{L}^{2}(\mathbb{Q})}^{2}
\end{aligned}
$$

por lo tanto, $A^{-1}\|G\|_{\mathbb{L}^{2}(\mathbb{Q})}^{2} \leq\left\|W^{-1} G\right\|_{\mathbb{L}^{2}(\mathfrak{Q})}^{2} \leq B^{-1}\|G\|_{\mathbb{L}^{2}(0)}^{2}$. con lo cual se obtiene lo deseado . 
De esta manera se tiene que $W$ es un isomorfismo topológico .

Afirmación 2: $W E_{m, n}(t, w)=Z g_{m b, n a}(t, w)$

En efecto, tomemos en $\mathbb{L}^{2}(\mathbb{Q})$ la base base ortonormal $\left\{E_{m, n}(t, w)\right\}$, entonces

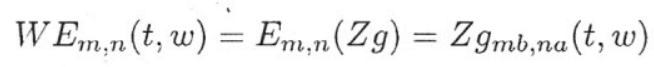

esto es por definición de $W$ y $Z\left(g_{m b, n a}\right)=E_{m, n}(Z g)$.

Por lo tanto $\left\{Z g_{m b, n a}(t, w)\right\}_{m, n \in \mathbb{Z}}$ es una base de Riesz para $\mathbb{L}^{2}(\mathbb{Q})$, si y sólo si, $\left\{g_{m b, n a}\right\}$ es una base de Riesz en $\mathbb{L}^{2}(\mathbb{R})$ o equivalentemente $\left\{g_{m b, n a}\right\}$ es un frame exacto para $\mathbb{L}^{2}(\mathbb{R})$, puesto que $\mathbb{L}^{2}(\mathbb{R})$ es un espacio de Hilbert Separable.

(iii) $\Rightarrow$ (ii) Inmediato. Ahora probaremos que

$$
C_{m, n}=\int_{0}^{1} \int_{0}^{1} \frac{(Z f)(t, w)}{(Z g)(t, w)} e^{-2 \pi i m t} e^{-2 \pi i n w} d t d w
$$

En efecto,

Afirmación 3: $Z(S f)=(Z f)|Z g|^{2}: \forall f \in \mathbb{L}^{2}(\mathbb{R})$, donde $S$ es el operador frame con respecto al frame $\left\{g_{m, b, n a}\right\}$.

En efecto,

$$
S f=\sum_{m, n}\left\langle f, g_{m b, n a}\right\rangle g_{m b, n a}
$$

entonces

$$
\begin{aligned}
Z(S f) & =\sum_{m, n}\left\langle f, g_{m b, n a}\right\rangle Z g_{m b, n a}=\sum_{m, n}\left\langle f, g_{m b, n a}\right\rangle E_{m, n}(Z g) \\
& =(Z g) \sum_{m, n}\left\langle f, g_{m b, n a}\right\rangle E_{m, n}=(Z g) \sum_{m, n}\left\langle Z f, Z g_{m b, n a}\right\rangle E_{m, n}
\end{aligned}
$$

pues la Transformada de Zak preserva norma y producto interno.

$$
\begin{aligned}
Z(S f) & =(Z g) \sum_{m, n}\left\langle Z f, E_{m, n}(Z g)\right\rangle_{\mathbb{L}^{2}(\mathbb{Q})} E_{m, n} \\
& =(Z g) \sum_{m, n}\left\langle(Z f)(\overline{Z g}), E_{m, n}\right\rangle_{\mathbb{L}^{2}(\mathbb{Q})} E_{m, n}
\end{aligned}
$$

como

$$
\begin{aligned}
\left\langle Z f, E_{m, n}(Z g)\right\rangle_{\mathbb{L}^{2}(Q)} & =\int_{0}^{1} \int_{0}^{1}(Z f)\left(\overline{E_{m, n}(Z g)}\right) \\
& =\int_{0}^{1} \int_{0}^{1}(Z f)(\overline{Z g}) \overline{E_{m . n}} \\
& =\left\langle(Z f)(\overline{Z g}), E_{m, n}\right\rangle \\
& =(Z g)(Z f)(\overline{Z g})=(Z f)|Z g|^{2}
\end{aligned}
$$

debido a que $\left\{E_{m, n}\right\}$ es una base ortonormal para $\mathbb{L}^{2}(\mathbb{Q})$.

Ahora reemplazando $f$ por $S^{-1} f$ en $Z(S f)=(Z f)|Z g|^{2}$, se tiene que :

$$
\begin{aligned}
Z\left(S S^{-1} f\right) & =\left(Z S^{-1} f\right)|Z g|^{2} \\
Z f & =\left(Z S^{-1} f\right)|Z g|^{2}
\end{aligned}
$$

luego

$$
Z\left(S^{-1} f\right)=\frac{Z f}{|Z g|^{2}}
$$

En particular, tomando $f=g$ en la relación (1), obtenemos

$$
Z\left(S^{-1} g\right)=\frac{Z g}{|Z g|^{2}}=\frac{1}{\overline{Z g}}
$$


Por lo tanto

$$
Z \widetilde{g}=\frac{1}{\overline{Z g}}
$$

donde

$$
\widetilde{g}=S^{-1} g \text { es el dual de } g .
$$

Finalmente para todo $f \in \mathbb{L}^{2}(\mathbb{R}), f=\sum_{m, n}\left\langle f, S^{-1} g_{m b, n a}\right\rangle g_{m b, n a}$, entonces

$$
\begin{aligned}
C_{m, n} & =\left\langle f, S^{-1} g_{m b, n a}\right\rangle=\left\langle f, \widetilde{g}_{m b, n a}\right\rangle=\left\langle Z f, Z \widetilde{g}_{m b, n a}\right\rangle \\
& =\left\langle Z f,\left(E_{m, n}\right)(Z \widetilde{g})\right\rangle=\left\langle Z f, E_{m, n}\left(\frac{1}{\overline{Z g}}\right)\right\rangle \\
& =\left\langle\frac{Z f}{Z g}, E_{m, n}\right\rangle_{\mathbb{L}^{2}(\mathbb{Q})} \\
& =\int_{0}^{1} \int_{0}^{1} \frac{Z f(t, w)}{Z_{g(t, w)}} \overline{E_{m, n}(t, w)} d t d w .
\end{aligned}
$$

Por lo tanto

$$
C_{m, n}=\int_{0}^{1} \int_{0}^{1} \frac{Z f(t, w)}{Z g(t, w)} e^{-2 \pi i m t} e^{-2 \pi i n w} d t d w
$$

\section{Observación:}

1.- La relación

$$
\begin{aligned}
\left\langle Z g_{k, b . l a}, Z g_{m b, n a}\right\rangle & =\int_{0}^{1} \int_{0}^{1} Z g_{k: b, l a} \overline{Z g}_{m b, n a} d t d w=\int_{0}^{1} \int_{0}^{1} E_{k, l}(t, w) Z g \bar{E}_{m, n}(t, w) \overline{Z g} d t d w \\
& =\int_{0}^{1} \int_{0}^{1} E_{k, l}(t, w) \bar{E}_{m, n}(t, w)|Z g|^{2} d t d w
\end{aligned}
$$

implica que $\left\{Z g_{m b, n a}\right\}$ ( y consecuentemente $\left\{g_{m b, n a}\right\}$ ), es ortonormal si y sólo si $|Z g|=1$, en casi todo punto.

En efecto, si $\left\{Z g_{m b, n a}\right\}$ es ortonormal, esto es $\left\{Z g_{m b, n a}\right\}$ es ortogonal y $\left\|Z g_{m b, n a}\right\|=1=\|Z g\|$, entonces $k=m, l=n$, luego

$$
\begin{aligned}
\left\langle Z g_{k, b, l a}, Z g_{m b, n a}\right\rangle & =\left\|Z g_{m b, n a}\right\|=1=\int_{0}^{1} \int_{0}^{1}\left|E_{m, n}(t, w)\right|^{2}|Z g|^{2} d t d w \\
1 & =\int_{0}^{1} \int_{0}^{1}|Z g|^{2} d t d w .
\end{aligned}
$$

Por lo tanto, $\left|Z_{g}\right|=1$ en casi todo punto.

Reciprocamente, si $|Z g|=1$ en casi todo punto, entonces

$$
\int_{0}^{1} \int_{0}^{1} E_{k \cdot l}(t, w) \bar{E}_{m . n}(t, w)|Z g|^{2} d t d w=\int_{0}^{1} \int_{0}^{1} E_{k: l}(t, w) \overline{E_{m, n}(t, w)} d t d w,
$$

Pero. $\left\{E_{m, n}\right\}$ es una base ortonormal. para $\mathbb{L}^{2}(\mathbb{Q})$, entonces

$$
\int_{0}^{1} \int_{0}^{1} E_{k, l}(t, w) \overline{E_{m, n}(t, u)} d t d w=\int_{0}^{1} \int_{0}^{1}\left|E_{m, n}\left(t, u^{\prime}\right)\right|^{2} d t \cdot d w=\int_{0}^{1} \int_{0}^{1} d t d w=1
$$

esto es

$$
\left\langle Z g_{m b, n a}, Z g_{m b, n a}\right\rangle=1=\left\|Z g_{m b, n a}\right\| .
$$

Además. $\left\{g_{m b, n a}\right\}$ es completa si y sólo si $(Z g) \neq 0$ en casi todo punto.

En efecto

$$
\left\langle f, g_{m b, n a}\right\rangle=\left\langle Z f, Z g_{m, b, n a}\right\rangle=\left\langle(Z f)(\overline{Z g}), E_{m, n}\right\rangle
$$

Supongamos que $\left\langle f, g_{m b, n a}\right\rangle=0$, para todo $m, n \in \mathbb{Z}$, esto es

$$
\left\langle f, g_{m b, n a}\right\rangle=\left\langle(Z f)(\overline{Z g}), E_{m, n}\right\rangle=0
$$


y como $\left\{E_{m, n}\right\}$ es un frame (por ser una base ortonormal) entonces $(Z f)(\overline{Z g})=0$, y como $Z g \neq 0$ en casi todo punto, se tiene que $Z f=0$, luego $f=0$. Por lo tanto $\left\{g_{m b, n a}\right\}$ es completa.

2.- La anterior observación indica que si $g$ es una función que es regular o de decaimiento muy rápido, esta no puede generar un frame cuando $a b=1$. Esto queda establecido precisamente en el siguiente teorema.

\section{Teorema 7 (Balian-Low).}

Sea $g \in \mathbb{L}^{2}(\mathbb{R})$ y $a, b>0$ con $a b=1$. Si $g$ genera un frame para $\mathbb{L}^{2}(\mathbb{R})$, entonces $\int x^{2}|g(x)|^{2} d x=\infty$ ó $\int \xi^{2}|\widehat{g}(\xi)|^{2} d \xi=\infty$ es decir $x g(x)$ ó $\xi \widehat{g}(\xi)$ no estan en $\mathbb{L}^{2}(\mathbb{R})$.

\section{Demostración.}

Supongamos que $\left\{g_{m, n}\right\}$ es un frame. Se tiene que

$$
\sum_{m, n}\left|\left\langle f, g_{m, n}\right\rangle\right|^{2}=\int_{0}^{1} \int_{0}^{1}|Z f(s, t)|^{2}|Z g(s, t)|^{2} d t d s
$$

y como $Z$ es unitario, entonces

$$
0<A \leq|Z g(s, t)|^{2} \leq B<\infty .
$$

Los vectores frames duales $\widetilde{g}_{m, n}$ estan dados por $\widetilde{g}_{m, n}=\left(F^{*} F\right)^{-1} g_{m, n}$. Luego desde que $Z\left(F^{*} F\right) Z^{-1}$ es igual a la multplicación por $|Z g|^{2}$, se deduce que

$$
\begin{aligned}
Z \widetilde{g}_{m, n} & =|Z g|^{-2} Z g_{m, n} \quad \text { ó } \\
Z \widetilde{g}_{m, n}(s, t) & =|Z g(s, t)|^{-2} e^{2 \pi i m s s} e^{-2 \pi i t n}(Z g)(s, t) \\
& =e^{2 \pi i m s s} e^{-2 \pi i t n}[\overline{Z g(s, t)}]^{-1}
\end{aligned}
$$

de esto se tiene

$$
\widetilde{g}_{m, n}(x)=e^{2 \pi i m x} \widetilde{g}(x-n), \operatorname{con} Z \widetilde{g}=\frac{1}{\overline{Z g}} .
$$

Ahora supongamos que $\int x^{2}|g(x)|^{2} d x<\infty \dot{o} \int \xi^{2}|\widehat{g}(\xi)|^{2} d \xi<\infty$, es decir que $Q g, \quad P g \in L^{2}(\mathbb{R})$, entonces se tiene que $\partial_{s}(Z g), \partial_{t}(Z g) \in L^{2}\left([0,1]^{2}\right)$.

Por consiguiente

$$
\begin{aligned}
\partial_{s} Z \widetilde{g} & =(\overline{Z g})^{-2} \overline{\partial_{s} Z g} \quad \mathrm{y} \\
\partial_{t} Z \widetilde{g} & =(\overline{Z g})^{-2} \overline{\partial_{t} Z g}
\end{aligned}
$$

estan en $L^{2}\left([0,1]^{2}\right)$; por lo tanto $Q \widetilde{g}, P \widetilde{g} \in L^{2}(\mathbb{R})$.

Se tiene que

$$
\begin{aligned}
\left\langle\widetilde{y}, g_{m, n}\right\rangle & =\left\langle Z \widetilde{y}, Z g_{m, n}\right\rangle \\
& =\int_{0}^{1} \int_{0}^{1} Z \widetilde{y}(s, t) \overline{Z g(s, t)} e^{-2 \pi i m . s} e^{2 \pi i t n} d t d s \\
& =\delta_{m 0} \delta_{n 0}
\end{aligned}
$$

de manera análoga se tiene

$$
\left\langle g, \widetilde{g}_{m, n}\right\rangle=\delta_{m 0} \delta_{n 0}
$$

Como $Q g, P \widetilde{g} \in L^{2}(\mathbb{R})$ y $\left\{g_{m, n}\right\}, \quad\left\{\widetilde{g}_{m, n}\right\}$ constituyen frames duales, se tiene

$$
\langle Q g, P \widetilde{g}\rangle=\sum_{m, n}\left\langle Q g, \widetilde{g}_{m, n}\right\rangle\left\langle g_{m, n}, P \widetilde{g}\right\rangle
$$

pero

$$
\begin{aligned}
\left\langle Q g, \tilde{g}_{m, n}\right\rangle & =\int x g(x) e^{-2 \pi i m x} \overline{\widetilde{g}(x-n)} d x \\
& =\left\langle g_{-m,-n} \cdot Q \tilde{g}\right\rangle, \text { esto debido a que }\left\langle g, \tilde{g}_{m, n}\right\rangle=\delta_{m 0} \delta_{n 0}
\end{aligned}
$$


de manera análoga

$$
\left\langle g_{m, n}, P \widetilde{g}\right\rangle=\left\langle P g, \widetilde{g}_{-m,-n}\right\rangle
$$

por consiguiente

$$
\langle Q g, P \widetilde{g}\rangle=\sum_{m, n}\left\langle P g, \widetilde{g}_{-m,-n}\right\rangle\left\langle g_{-m,-n}, Q \widetilde{g}\right\rangle=\langle P g, Q \widetilde{g}\rangle
$$

donde el último término esta bien definido pues $P g, Q \widetilde{g} \in L^{2}(\mathbb{R})$.

Pero $\langle Q g, P \widetilde{g}\rangle=\langle P g, Q \widetilde{g}\rangle$, es una contradicción, veamos sean dos funciones $f_{1}, f_{2}$ satisfaciendo

$$
\begin{aligned}
& \left|f_{j}(x)\right| \leq C\left(1+x^{2}\right)^{-1}, \mathrm{y} \\
& \left|\widetilde{f}_{j}(\xi)\right| \leq C\left(1+\xi^{2}\right)^{-1}
\end{aligned}
$$

entonces se tiene

$$
\begin{aligned}
\left\langle Q f_{1}, P f_{2}\right\rangle & =\int x f_{1}(x) i \overline{f_{2}^{\prime}(x)} d x \\
& =-\int i\left[x f_{1}(x)+f_{1}(x)\right] f_{2}(x) d x \\
& =-i\left\langle f_{1}, f_{2}\right\rangle+\left\langle P f_{1}, Q f_{2}\right\rangle .
\end{aligned}
$$

Por otro lado, desde que $P g, Q g \in L^{2}(\mathbb{R})$, existen $g_{n}$ satisfaciendo

$$
\begin{aligned}
& \left|g_{n}(x)\right| \leq C_{n}\left(1+x^{2}\right)^{-1}, \mathrm{y} \\
& \left|\widetilde{g}_{n}(\xi)\right| \leq C_{n}\left(1+\xi^{2}\right)^{-1}
\end{aligned}
$$

tal que $\lim _{n \rightarrow \infty} g_{n}=g, \lim _{n \rightarrow \infty} P g_{n}=P g, \lim _{n \rightarrow \infty} Q g_{n}=Q g$ (se puede tomar por ejemplo $g_{n}=\sum_{k=0}^{n}\left\langle g, H_{k}\right\rangle H_{k}$, donde $H_{k}$ son las funciones de Hermite). Una similar sucesión $\widetilde{g}_{n}$ puede ser construida para $g_{n}$, entonces

$$
\begin{aligned}
\langle P g, Q \widetilde{g}\rangle & =\lim _{n \rightarrow \infty}\left\langle P g_{n}, Q \widetilde{g_{n}}\right\rangle \\
& =\lim _{n \rightarrow \infty}\left[\left\langle Q g_{n}, P \widetilde{g_{n}}\right\rangle+i\left\langle g_{n}, \widetilde{g_{n}}\right\rangle\right] \\
& =\langle Q g, P \widetilde{g}\rangle+i\langle g, \widetilde{g}\rangle
\end{aligned}
$$

pero como $\langle Q g, P \widetilde{g}\rangle=\langle P g, Q \widetilde{g}\rangle$, entonces $\langle g, \widetilde{g}\rangle=0$ lo cual es mua contradicción pucsto que $\langle g, \widetilde{g}\rangle=1$.

\section{Conclusiones}

Resumiendo los resultados anteriores; para $g \in \mathbb{L}^{2}(\mathbb{R})$ se tiene que:

i) Si $a b>1$, es imposible que $\left\{g_{m b, n a}\right\}$ sea un frame para $\mathbb{L}^{2}(\mathbb{R})$.

ii) Si $a b=1$, entonces $\left\{g_{m b, n a}\right\}$ puede ser un frame para $\mathbb{L}^{2}(\mathbb{R})$, pero $g$ no puede ser una función regular o de decaimiento muy rápido.

iii) Si $0<a b<1$, entonces $\left\{g_{m b, n a}\right\}$ puede ser un frane para $\mathbb{L}^{2}(\mathbb{R})$, con una buena ()ndícula madre: para $g$. De hecho, g puede ser infinitanente diferenciable con soporte compacto. 


\section{Bibliografía}

[1] BEYLKIN G., COIFMAN R., ROKHLIN V. (1991), Fast Wavelet Transforms and numerical algorithms, Comm. Pure and Appl. Math., 44, pp.141-183.

[2] BURKE-HUBBAR, B. (1995), Ondes et Ondelettes, pour la science, París.

[3] CHUI, C. (1992), An Introduction to Wavelets. Academic Press, Boston.

[4] CHUI, C., Wavelets: a Tutorial in Theory and Applications. Academic Press, Boston, (1992).

[5] COHEN A., DAUBECHIES I.. \& Vial, P. (1993), Wavelets on the interval and fast wavelet transform, Journal of Applied and Computational Harmonic Analysis 1, pp. 54-81.

[6] DAUBECHIES I. (1922): Ten lectures on Wavelets, CBMS-NSF Regional Conference Series in Applied Mathematics, SIAM Publ. Philadelphia.

[7] DAUBECHIES I. (1988): Orthonormal bases of Compactly Supported Wavelets, Comm. Pure and Appl. Math. 41, pp. 909-996.

[8] LOKENATH D. (2002), Wavelets transforms and their Applications.Birkhäuser, Boston. 